\title{
A retrospective, observational, real-world evidence study to understand the clinical and obstetric characteristics and tolerability profile of women in labor managed with camylofin dihydrochloride injection
}

\author{
Vinita K. Taneja ${ }^{1 *}$, Milind A. Telang ${ }^{2}$
}

${ }^{1}$ Amrit Child Care and Maternity Home, Agra, Uttar Pradesh, India

2Indira Maternity Home, Pune, Maharashtra, India

Received: 24 November 2020

Accepted: 04 February 2021

*Correspondence:

Dr. Vinita K. Taneja,

E-mail: vinilively16@gmail.com

Copyright: (C) the author(s), publisher and licensee Medip Academy. This is an open-access article distributed under the terms of the Creative Commons Attribution Non-Commercial License, which permits unrestricted non-commercial use, distribution, and reproduction in any medium, provided the original work is properly cited.

\begin{abstract}
Background: To determine the clinical and obstetric characteristics and tolerability profile of pregnant women in the active phase of labor who were managed with Camylofin dihydrochloride injection.

Methods: Retrospective data of 210 full-term pregnant women in the active phase of labor who were managed with Camylofin injection doses of $50 \mathrm{mg}, 50+25 \mathrm{mg}$ and $50+50 \mathrm{mg}$ were considered in the study. The comparative effectiveness of 3 doses was evaluated using independent T-test and ANOVA at 5\% level of significance.

Results: The overall mean maternal age was $25.4( \pm 4.14)$ years with mean gestational age of $38.8( \pm 1.04)$ weeks. In total, $77.1 \%(\mathrm{n}=162)$ of patients needed $50 \mathrm{mg}$ dose of Camylofin injection for adequate cervical dilatation, and additional doses of $25 \mathrm{mg}$ or $50 \mathrm{mg}$ were required in $16.7 \%(\mathrm{n}=35)$ and $6.2 \%(\mathrm{n}=13)$, of patients, respectively to accelerate the labor. The mean cervical dilatation rates increasing dose viz $2.8 \mathrm{~cm} / \mathrm{hr}, 4.1 \mathrm{~cm} / \mathrm{hr}$ and $2.9 \mathrm{~cm} / \mathrm{hr}$, respectively. The adverse events were $14.8 \%(n=23)$ in $50 \mathrm{mg}$ arm, 34.3\% $(n=12)$ in $50+25$ arm and $69.0 \%(n=9)$ in 50+50 arm. Of the total, 34 events $(50.0 \%)$ were related to Camylofin dihydrochloride injection, i.e. ADRs.

The means of cervical dilation rate, active phase duration and total duration of labor were statistically significant (pvalue $<0.001$ ) for $50 \mathrm{mg}$ versus $50+25 \mathrm{mg}$.

Conclusions: The study infers Camylofin injection usage with consideration of dose escalation in the routine clinical management of labor. Both mother and neonate had shown comparable tolerability profile across the three dosage arms with minimal events.
\end{abstract}

Keywords: Real-world evidence, Camylofin, Labor management, Patient outcomes

\section{INTRODUCTION}

Labor is a multifactorial process that involves myometrial contraction, cervical effacement and dilatation and the expulsion of the fetus and placenta in an orderly manner. ${ }^{1}$ The progress of labor is assessed by progressive dilatation and effacement of the cervix and the descent of the presenting part. $^{2}$ Normal labor has been defined as when an infant is born within 12 hours of active labor. ${ }^{3}$ The most vexing issue faced by obstetricians is the deviation of normal labor.
Prolonged (obstructed) labor affects 3-6\% of women in labor globally4 and contributes to an estimated $2.8 \%$ of maternal mortality worldwide. ${ }^{5,6}$ In developing countries, it ranges from 4 to $70 \%$ of all maternal deaths along with high prenatal mortality rate. ${ }^{4}$ Obstructed labor is seen between $0.56-1.89 \%$ of all deliveries in the referral hospitals of India. ${ }^{7}$ Recent studies from India have reported the incidence of prolonged pregnancies were anywhere from $4.62 \% 8$ to $8.9 \%{ }^{4}$ 
Active management of labor is now a part of modern obstetrics and has shown to decrease the occurrence of prolonged labor when compared to physiological or expectant management. ${ }^{9}$ A Cochrane review found that active management significantly shortened the duration of labor by 1.27 hours, while the first stage of labor was significantly reduced by 1.56 hours. ${ }^{10}$ Interventions to shorten labor, such as antispasmodics, can be used as a treatment strategy in order to decrease the incidence of prolonged labor.

Antispasmodics are drugs that have either musculotropic or neurotropic effects and are used to relieve spasms of smooth muscle tissue. ${ }^{9}$ Smooth muscle constitutes about $15 \%$ of the cervix, which is mainly found just below the internal OS. ${ }^{11,12}$ Administering antispasmodics during labor could lead to faster and more effective dilatation of the cervix. ${ }^{13}$

Camylofin dihydrochloride a selective phosphodiesterase (PDE-4) enzyme inhibitor, has both musculotropic and neurotropic effects and has been recommended for use in acceleration of labor for more than six decades. ${ }^{14}$ Camylofin dihydrochloride primarily acts on the smooth muscles (intestine, ureter and cervix). It is a preferential cervical dilator, with no interference on the uterine contractions. In addition, its pharmacological effect on glands, eyes, heart, and circulation is minimal. ${ }^{14}$ Moreover, Camylofin dihydrochloride does not interfere with uterine contractility due to its PDE-4 iso-enzyme selectivity. Owing to this preferential cervical dilating action, Camylofin dihydrochloride has been widely recommended for use in accelerating the first stage of labor. $^{14}$

There is a growing body of evidence to support the effectiveness and tolerability of Camylofin dihydrochloride in the active management of labor. ${ }^{1,14-17}$ Camylofin dihydrochloride has a fast onset and a long duration of action, coupled with superior effectiveness compared to other agents like Drotaverine, Hyoscine, and Valethemate. ${ }^{14}$ The results from various studies have demonstrated its effectiveness in the acceleration of labor in young women, both primigravida, and multigravida. ${ }^{14}$ Camylofin has been in use for over 60 years, 18,19 with multiple citations in academic textbooks of repute,27-32 treatment protocol and multiple research publications. ${ }^{14,26,27}$ However, there is a lack of consensus on appropriate usage of the dosage regimen of Camylofin dihydrochloride injection in the acceleration of labor.

The present multicenter, retrospective real-world evidence study was designed to understand the clinical and obstetric characteristics and tolerability profile of women in labor managed with three doses $(50,50+25$ and $50+50 \mathrm{mg}$ ) Camylofin dihydrochloride injection. Additionally, was also evaluated the comparative effectiveness of the three dose regimens of Camylofin dihydrochloride injection in terms of duration of active phase, cervical dilatation rate and total duration of labor across primigravida and multigravida women.

\section{METHODS}

This was a retrospective, observational, real-world evidence multicenter study designed to understand clinical and obstetric characteristics of pregnant women in the active phase of labor who were managed with Camylofin dihydrochloride injection in a real-world clinical practice setting. There was no prospective assessment or study specific procedures with patients during the study. The treatment and collection of the data were according to the real-world clinical practice.

The study was conducted in conformity with the principles of the declaration of Helsinki, international council for harmonization-good clinical practices (ICH-GCP) guidelines, Indian council of medical research, Indian GCP guidelines, and as per the approved protocol. The process of data analysis was only initiated after approval from the independent ethics committee. Since this was a retrospective data collection study, informed consent (ICF) was not required and accordingly, ICF waiver approval was taken from the ethics committee. Patient confidentiality was maintained during the data entry and analysis process.

The pregnant women who were previously managed with Camylofin dihydrochloride injection either with single dose $50 \mathrm{mg}$ or an additional dose of $25 \mathrm{mg}(50+25 \mathrm{mg})$ or $50 \mathrm{mg}(50+50 \mathrm{mg})$ during the labor time were considered for the study. A time gap 1-hour interval between the two doses $(50+25 \mathrm{mg}$ and $50+50 \mathrm{mg})$ of Camylofin dihydrochloride injections was maintained during the management. The decision to escalate the dose was entirely to the clinical discretion of the investigators. The data of pregnant women treated in the period of five years, starting from May 2014 to June 2019 was considered for this retrospective analysis. The study was conducted at two Indian centers, Agra, Uttar Pradesh and Pune, Maharashtra including 105 pregnant women from each center.

The inclusive criteria was all adult pregnant women (gestational age 37 to 40 weeks) in labor who were managed with Camylofin injection. The exclusion criteria were women with multiple pregnancies, any comorbidities or condition managed with antihistaminics, tricyclic antidepressants, phenothiazines, disopyramide, pethidine having drug interaction with Camylofin and patients with incomplete hospital records.

The following data at respective antenatal (ANC) visits was collected -

Baseline data from ANC visit - The data collated for treatment initiation visit consisted of demographic details (age, weight, height), past pregnancy details (gravidity, parity, history of previous deliveries), and expected date of delivery. 
ANC follow-up visits - data collected in follow-up visits comprised details of first trimester, second and third trimester, presence of any complications, general and systemic examinations, abdomen inspection, palpation, history of comorbidities and medications.

Intrapartum - data during labor included gestational age at admission, time of onset of labor, medication history, details of dosage, frequency and time of Camylofin dihydrochloride injection administered, and recorded cervical dilatation, cervical effacement, uterine contractions and fetal characteristics (heart rate, Apgar score), at regular time intervals. In addition, mode of delivery, any maternal and fetal complications, neonatal condition at birth i.e. birth weight and Apgar score at 1 minute and 5 minutes were also noted.

Following parameters were calculated from the base parameters for every patient: duration of active phase of (1st stage of) labor, duration of 2nd and 3rd stage of labor and total duration of labor.

Postpartum - safety data captured in the form of reported adverse events (AEs) and adverse drug reaction (ADRs) for both maternal and neonatal, post administration of Camylofin injection.

The evidence of effectiveness was evaluated based on the comparative effectiveness of different dose regimens of Camylofin dihydrochloride injection between primigravida and multigravida patients. While the tolerability parameters were from the reported ADRs and AEs across different doses of Camylofin dihydrochloride injection.

\section{Statistical analysis}

The categorical data was presented as percentage distribution (e.g., primigravida, multigravida). In the case of continuous parameters, calculation of mean $( \pm \mathrm{SD})$, median, minimum and maximum (e.g., age, weight, gravidity, parity, gestational age on admission, rate of cervical dilatation and cervical effacement, and fetal characteristics (Apgar score)) was done. The same analysis was repeated for patients managed with three dosages of Camylofin dihydrochloride injection. The frequency count for each dosage arm was calculated. For assessment of safety and tolerability, the proportion of patients reported AEs or ADRs and a list of these events (serious or nonserious), were described in terms of number and percentage of patients (n (\%)) for each event. The causality analysis of ADRs as reported by the site PIs was presented in number and percentages.

The comparative assessment of mean active phase of the first stage of labor, mean cervical dilatation rate and mean total duration of labor was done for primigravida and multigravida patients (for three different dose regimes of Camylofin dihydrochloride injection), using independent sample t-test (to compare the duration between the two groups among various doses) and ANOVA at 5\% level of significance and the corresponding p-values were presented.

\section{RESULTS}

\section{Clinical and obstetric characteristics}

The baseline clinical and obstetric characteristics of all enrolled patients are summarized Camylofin doses viz. in Table 1. The maternal characteristics including maternal age, weight, gravidity, parity, gestational age, cervical dilatation and cervical effacement and fetal characteristics comprising APGAR score. The parameters are detailed in terms of count $(\mathrm{N})$, mean $( \pm \mathrm{SD})$, median, minimum and maximum values.

Table 1: Summary of clinical and obstetric characteristics of study populations is given dose viz (n=210).

\begin{tabular}{|c|c|c|c|c|c|c|c|c|}
\hline Category & \multicolumn{2}{|l|}{ Variable } & $\mathbf{N}(\%)$ & Mean & \pm SD & Median & Min & Max \\
\hline \multirow{13}{*}{$\begin{array}{l}\text { Camylofin } \\
\text { Injection } 50 \mathrm{mg}\end{array}$} & \multicolumn{2}{|c|}{ Maternal age (in years) } & $162(77.1)$ & 25.4 & 4.33 & 25.0 & 18.0 & 38.0 \\
\hline & \multicolumn{2}{|c|}{ Weight (in kgs) } & $162(77.1)$ & 63.3 & 6.56 & 63.0 & 48.0 & 86.0 \\
\hline & \multirow{3}{*}{ Gravidity } & Primigravida & $69(42.6)$ & 1.0 & 0.00 & 1.0 & 1.0 & 1.0 \\
\hline & & Multigravida & $93(57.4)$ & 2.5 & 0.80 & 2.0 & 2.0 & 7.0 \\
\hline & & Total & $162(77.1)$ & 1.9 & 0.97 & 2.0 & 1.0 & 7.0 \\
\hline & \multicolumn{2}{|l|}{ Parity } & $162(77.1)$ & 1.3 & 0.83 & 1.0 & 0.0 & 3.0 \\
\hline & \multicolumn{2}{|c|}{ Gestational age (in weeks) } & $162(77.1)$ & 39.0 & 1.06 & 39.0 & 37.0 & 40.0 \\
\hline & \multicolumn{2}{|c|}{$\begin{array}{l}\text { Cervical dilatation rate } \\
\text { (cm per hour) }\end{array}$} & $162(77.1)$ & 2.8 & 1.55 & 2.4 & 0.3 & 9.3 \\
\hline & \multicolumn{2}{|c|}{$\begin{array}{l}\text { Cervical effacement rate ( } \% \\
\text { per hour) }\end{array}$} & $162(77.1)$ & 18.0 & 10.96 & 15.2 & 0.0 & 66.7 \\
\hline & \multicolumn{2}{|c|}{ APGAR Score at $1 \mathrm{~min}$} & $162(77.1)$ & 8.6 & 1.10 & 8.0 & 6.0 & 10.0 \\
\hline & \multicolumn{2}{|c|}{ APGAR Score at $5 \mathrm{~min}$} & $162(77.1)$ & 9.3 & 0.53 & 9.0 & 8.0 & 10.0 \\
\hline & \multicolumn{2}{|c|}{ Maternal age (in years) } & $35(16.7)$ & 25.0 & 3.13 & 25.0 & 19.0 & 33.0 \\
\hline & \multicolumn{2}{|c|}{ Weight (in kgs) } & $35(16.7)$ & 63.3 & 7.57 & 62.0 & 50.0 & 80.0 \\
\hline
\end{tabular}




\begin{tabular}{|c|c|c|c|c|c|c|c|c|}
\hline Category & Variable & & $\mathbf{N}(\%)$ & Mean & \pm SD & Median & Min & $\operatorname{Max}$ \\
\hline \multirow{9}{*}{$\begin{array}{l}\text { Camylofin } \\
\text { Injection } 75 \mathrm{mg} \\
(50+25)\end{array}$} & \multirow{3}{*}{ Gravidity } & Primigravida & $11(31.4)$ & 1.0 & 0.00 & 1.0 & 1.0 & 1.0 \\
\hline & & Multigravida & $24(68.6)$ & 2.3 & 0.48 & 2.0 & 2.0 & 3.0 \\
\hline & & Total & $35(16.7)$ & 1.9 & 0.74 & 2.0 & 1.0 & 3.0 \\
\hline & \multicolumn{2}{|l|}{ Parity } & 35 (16.7) & 0.7 & 0.75 & 1.0 & 0.0 & 2.0 \\
\hline & \multicolumn{2}{|c|}{ Gestational age (in weeks) } & $35(16.7)$ & 38.4 & 0.77 & 39.0 & 37.0 & 39.0 \\
\hline & \multicolumn{2}{|c|}{$\begin{array}{l}\text { Cervical dilatation rate } \\
(\mathrm{cm} \text { per hour })\end{array}$} & $35(16.7)$ & 4.1 & 1.14 & 4.4 & 1.7 & 7.0 \\
\hline & \multicolumn{2}{|c|}{$\begin{array}{l}\text { Cervical effacement rate (\% } \\
\text { per hour) }\end{array}$} & $35(16.7)$ & 27.1 & 9.01 & 29.6 & 0.0 & 50.0 \\
\hline & \multicolumn{2}{|c|}{ APGAR Score at $1 \mathrm{~min}$} & $35(16.7)$ & 9.9 & 0.47 & 10.0 & 8.0 & 10.0 \\
\hline & \multicolumn{2}{|c|}{ APGAR Score at $5 \mathrm{~min}$} & $35(16.7)$ & 9.9 & 0.24 & 10.0 & 9.0 & 10.0 \\
\hline \multirow{11}{*}{$\begin{array}{l}\text { Camylofin } \\
\text { Injection } 100 \mathrm{mg} \\
(\mathbf{5 0 + 5 0 )}\end{array}$} & \multicolumn{2}{|c|}{ Maternal age (in years) } & $13(6.2)$ & 26.1 & 4.21 & 27.0 & 19.0 & 35.0 \\
\hline & \multicolumn{2}{|c|}{ Weight (in kgs) } & $13(6.2)$ & 66.2 & 6.00 & 65.0 & 55.0 & 75.0 \\
\hline & \multirow{3}{*}{ Gravidity } & Primigravida & $0(0.0)$ & 0.0 & 0.0 & 0.0 & 0.0 & 0.0 \\
\hline & & Multigravida & $13(100)$ & 2.2 & 0.44 & 2.0 & 2.0 & 3.0 \\
\hline & & Total & $13(6.2)$ & 2.2 & 0.44 & 2.0 & 2.0 & 3.0 \\
\hline & \multicolumn{2}{|l|}{ Parity } & $13(6.2)$ & 0.7 & 0.63 & 1.0 & 0.0 & 2.0 \\
\hline & \multicolumn{2}{|c|}{ Gestational age (in weeks) } & $13(100)$ & 38.5 & 1.13 & 38.0 & 37.0 & 40.0 \\
\hline & \multicolumn{2}{|c|}{$\begin{array}{l}\text { Cervical dilatation rate } \\
(\mathrm{cm} \text { per hour) }\end{array}$} & $13(6.2)$ & 2.9 & 0.35 & 2.8 & 2.3 & 3.5 \\
\hline & \multicolumn{2}{|c|}{$\begin{array}{l}\text { Cervical effacement rate (\% } \\
\text { per hour) }\end{array}$} & $13(6.2)$ & 27.1 & 6.63 & 23.1 & 20.0 & 38.5 \\
\hline & \multicolumn{2}{|c|}{ APGAR Score at $1 \mathrm{~min}$} & $13(6.2)$ & 10.0 & 0.00 & 10.0 & 10.0 & 10.0 \\
\hline & \multicolumn{2}{|c|}{ APGAR Score at $5 \mathrm{~min}$} & $13(6.2)$ & 10.0 & 0.00 & 10.0 & 10.0 & 10.0 \\
\hline \multirow{11}{*}{$\begin{array}{l}\text { Camylofin } \\
\text { Injection Overall }\end{array}$} & \multicolumn{2}{|c|}{ Maternal age (in years) } & $210(100)$ & 25.4 & 4.14 & 25.0 & 18.0 & 38.0 \\
\hline & \multicolumn{2}{|c|}{ Weight (in kgs) } & $210(100)$ & 63.5 & 6.71 & 63.0 & 48.0 & 86.0 \\
\hline & \multirow{3}{*}{ Gravidity } & Primigravida & $80(38.1)$ & 1.0 & 0.00 & 1.0 & 1.0 & 1.0 \\
\hline & & Multigravida & $130(61.9)$ & 2.3 & 0.73 & 2.0 & 2.0 & 7.0 \\
\hline & & Total & $210(100)$ & 1.9 & 0.91 & 2.0 & 1.0 & 7.0 \\
\hline & \multicolumn{2}{|c|}{ Parity } & $210(100)$ & 1.2 & 0.84 & 1.0 & 0.0 & 3.0 \\
\hline & \multicolumn{2}{|c|}{ Gestational age (in weeks) } & $210(100)$ & 38.8 & 1.04 & 39.0 & 37.0 & 40.0 \\
\hline & \multicolumn{2}{|c|}{$\begin{array}{l}\text { Cervical dilatation rate } \\
\text { (cm per hour) }\end{array}$} & $210(100)$ & 3.0 & 1.53 & 2.8 & 0.3 & 9.3 \\
\hline & \multicolumn{2}{|c|}{$\begin{array}{l}\text { Cervical effacement rate (\% } \\
\text { per hour) }\end{array}$} & $210(100)$ & 20.1 & 11.09 & 20.0 & 0.0 & 66.7 \\
\hline & \multirow{2}{*}{\multicolumn{2}{|c|}{ APGAR Score at $1 \mathrm{~min}$}} & $210(100)$ & 8.9 & 1.12 & 9.0 & 6.0 & 10.0 \\
\hline & & & $210(100)$ & 9.5 & 0.54 & 10.0 & 8.0 & 10.0 \\
\hline
\end{tabular}

Of the total enrolled patients $(\mathrm{N}=210), 162$ patients (77.1\%) were given Camylofin injections $50 \mathrm{mg}$ dose, 35 patients $(16.7 \%)$ required additional injection of $25 \mathrm{mg}$ dose $(75 \mathrm{mg}(50+25))$ and 13 patients $(6.2 \%)$ needed additional injection of $50 \mathrm{mg}$ dose $(100 \mathrm{mg}(50+50))$ to manage the labor.
The maternal age of study population was distributed in a range from 18 to 38 years with overall mean of 25.4 $( \pm 4.14)$ years. The mean maternal weight was comparable between $50 \mathrm{mg}(63.3 \mathrm{kgs})$ and $75 \mathrm{mg}(50+25)(63.3 \mathrm{kgs})$ categories whereas in $100 \mathrm{mg}(50+50)$ category, the mean was slightly higher $(66.2 \mathrm{kgs})$.

Table 2: Overall group analysis.

\begin{tabular}{|c|c|c|c|c|}
\hline $\begin{array}{l}\text { Groups } \\
\text { considered }\end{array}$ & Parameter & $\begin{array}{l}\text { Active phase of } 1^{\text {st }} \text { stage } \\
\text { duration (in minutes) }\end{array}$ & $\begin{array}{l}\text { Cervical dilatation } \\
\text { rate (cm per hour) }\end{array}$ & $\begin{array}{l}\text { Total duration of } \\
\text { labor (in hours) }\end{array}$ \\
\hline \multirow{3}{*}{$\begin{array}{l}\text { Camylofin } \\
\text { Injection } \\
50 \mathrm{mg} \text { vs } \\
75 \mathrm{mg}(\mathbf{5 0}+\mathbf{2 5})\end{array}$} & $\begin{array}{l}50 \mathrm{mg}(\mathrm{N}=162) \\
\text { mean }( \pm \mathrm{SD})\end{array}$ & $167.5( \pm 96.32)$ & $2.8( \pm 1.55)$ & $3.1( \pm 2.47)$ \\
\hline & $\begin{array}{l}50+25(\mathrm{~N}=35) \\
\text { mean }( \pm \mathrm{SD})\end{array}$ & $84.6( \pm 37.75)$ & $4.1( \pm 1.14)$ & $1.8( \pm 0.56)$ \\
\hline & $\begin{array}{l}\text { Mean difference } \\
\text { 95\% C.I }\end{array}$ & $63.29-102.44$ & $-1.82--0.9$ & $0.84-1.69$ \\
\hline
\end{tabular}

Continued. 


\begin{tabular}{|c|c|c|c|c|}
\hline $\begin{array}{l}\text { Groups } \\
\text { considered }\end{array}$ & Parameter & $\begin{array}{l}\text { Active phase of } 1^{\text {st }} \text { stage } \\
\text { duration (in minutes) }\end{array}$ & $\begin{array}{l}\text { Cervical dilatation } \\
\text { rate (cm per hour) }\end{array}$ & $\begin{array}{l}\text { Total duration of } \\
\text { labor (in hours) }\end{array}$ \\
\hline & SE & 16.6 & 0.3 & 0.4 \\
\hline & $p$-value & $<0.0001$ & $<0.0001$ & $<0.0001$ \\
\hline \multirow{5}{*}{$\begin{array}{l}\text { Camylofin } \\
\text { Injection } \\
\mathbf{5 0} \text { mg vs } 100 \\
\text { mg }(\mathbf{5 0 + 5 0})\end{array}$} & $\begin{array}{l}50 \mathrm{mg}(\mathrm{N}=162) \\
\text { mean }( \pm \mathrm{SD})\end{array}$ & $167.5( \pm 96.32)$ & $2.8( \pm 1.55)$ & $3.1( \pm 2.47)$ \\
\hline & $\begin{array}{l}50+50(\mathrm{~N}=13) \text { mean } \\
( \pm \mathrm{SD})\end{array}$ & $118.8( \pm 19.59)$ & $2.8( \pm 0.35)$ & $2.5( \pm 0.30)$ \\
\hline & $\begin{array}{l}\text { Mean difference } \\
95 \% \text { C.I }\end{array}$ & $30.19-67.26$ & $-0.41-0.21$ & $0.20-1.03$ \\
\hline & SE & 26.8 & 0.4 & 0.7 \\
\hline & $p$-value & $<0.0001$ & 0.5349 & 0.0042 \\
\hline \multirow{5}{*}{$\begin{array}{l}\text { Camylofin } \\
\text { Injection } \\
75 \mathrm{mg}(50+25) \\
\text { vs } 100 \mathrm{mg} \\
(\mathbf{5 0 + 5 0 )}\end{array}$} & $\begin{array}{l}50+25(\mathrm{~N}=35) \\
\text { mean }( \pm \mathrm{SD})\end{array}$ & $84.6( \pm 37.75)$ & $4.1( \pm 1.14)$ & $1.8( \pm 0.56)$ \\
\hline & $\begin{array}{l}50+50(\mathrm{~N}=13) \text { mean } \\
( \pm \mathrm{SD})\end{array}$ & $118.8( \pm 19.59)$ & $2.8( \pm 0.35)$ & $2.5( \pm 0.30)$ \\
\hline & $\begin{array}{l}\text { Mean difference } \\
95 \% \text { C.I }\end{array}$ & $-51.07--17.21$ & $0.83-1.70$ & $-0.91--0.40$ \\
\hline & SE & 11.0 & 0.3 & 0.2 \\
\hline & p-value & 0.0002 & $<0.0001$ & $<0.0001$ \\
\hline
\end{tabular}

*The p-value and 95\% C.I were assessed using an independent t-test

Table 3: Tests of significance (ANOVA) for exploratory endpoints.

\begin{tabular}{|c|c|c|c|c|c|c|c|}
\hline \multirow[b]{2}{*}{$\begin{array}{l}\text { Camylofin } \\
\text { dose (mg) }\end{array}$} & \multirow[b]{2}{*}{ LS Mean } & \multirow[b]{2}{*}{ SE } & \multicolumn{2}{|c|}{$95 \%$ C.I } & \multicolumn{3}{|l|}{ p-value } \\
\hline & & & Lower & Upper & $\begin{array}{l}50 \text { vs } 75 \\
(50+25)\end{array}$ & $\begin{array}{l}75(50+25) \\
\text { vs } 100(50+50)\end{array}$ & $\begin{array}{l}50 \text { vs } 100 \\
(50+50)\end{array}$ \\
\hline \multicolumn{8}{|c|}{ Active phase of $1^{\text {st }}$ stage duration (in minutes) } \\
\hline 50 & $167.5^{\circ}$ & 6.8 & 154.10 & 180.88 & \multirow{3}{*}{$<0.0001$} & \multirow{3}{*}{0.4450} & \multirow{3}{*}{0.1260} \\
\hline $\begin{array}{l}75 \\
(50+25)\end{array}$ & 84.6 & 14.6 & 55.82 & 113.43 & & & \\
\hline $100(50+50)$ & 118.8 & 24.0 & 71.50 & 166.03 & & & \\
\hline Overall & 150.7 & 6.3 & 138.19 & 163.14 & $<0.0001$ & & \\
\hline \multicolumn{8}{|c|}{ Cervical dilatation rate (cm per hour) } \\
\hline 50 & 2.8 & 0.1 & 2.54 & 2.99 & \multirow{3}{*}{$<0.0001$} & \multirow{3}{*}{0.0214} & \multirow{3}{*}{0.9706} \\
\hline $\begin{array}{l}75 \\
(50+25)\end{array}$ & 4.1 & 0.2 & 3.64 & 4.60 & & & \\
\hline $100(50+50)$ & 2.9 & 0.4 & 2.07 & 3.65 & & & \\
\hline Overall & 3.0 & 0.1 & 2.54 & 3.15 & $<0.0001$ & & \\
\hline \multicolumn{8}{|c|}{ Total duration of labor (in hours) } \\
\hline 50 & 3.1 & 0.2 & 2.75 & 3.43 & \multirow{3}{*}{0.0063} & \multirow{4}{*}{0.6303} & \multirow{4}{*}{0.5968} \\
\hline $\begin{array}{l}75 \\
(50+25)\end{array}$ & 1.8 & 0.4 & 1.10 & 2.56 & & & \\
\hline $100(50+50)$ & 2.5 & 0.6 & 1.28 & 3.68 & & & \\
\hline Overall & 2.8 & 0.1 & 2.54 & 3.15 & 0.0077 & & \\
\hline
\end{tabular}

Table 4: Primigravida vs Multigravida within the treatment arms.

\begin{tabular}{|c|c|c|c|c|}
\hline Category & Statistical variable & $\begin{array}{l}\text { Active phase of } \\
1^{\text {st }} \text { stage duration } \\
\text { (in minutes) }\end{array}$ & $\begin{array}{l}\text { Cervical dilatation } \\
\text { rate } \\
\text { (cm per hour) }\end{array}$ & $\begin{array}{l}\text { Total duration of } \\
\text { labor } \\
\text { (in hours) }\end{array}$ \\
\hline \multirow{5}{*}{$\begin{array}{l}\text { Camylofin } \\
50 \mathrm{mg} \\
\text { Primigravida } \\
\text { Vs Multigravida }\end{array}$} & $\begin{array}{l}\text { Primigravida }(\mathrm{N}=69) \\
\text { Mean }( \pm \mathrm{SD})\end{array}$ & $201.4( \pm 100.24)$ & $2.3( \pm 1.16)$ & $3.4( \pm 2.07)$ \\
\hline & $\begin{array}{l}\text { Multigravida }(\mathrm{N}=93) \\
\text { Mean }( \pm \mathrm{SD})\end{array}$ & $142.3( \pm 85.48)$ & $3.1( \pm 1.71)$ & $2.9( \pm 2.72)$ \\
\hline & $\begin{array}{l}\text { Mean difference } 95 \% \\
\text { C.I }\end{array}$ & $29.46-88.69$ & $-1.29--0.40$ & $-0.20-1.29$ \\
\hline & SE & 14.6 & 0.2 & 0.4 \\
\hline & $p$ value & 0.0001 & 0.0002 & 0.1513 \\
\hline
\end{tabular}




\begin{tabular}{|c|c|c|c|c|}
\hline Category & Statistical variable & $\begin{array}{l}\text { Active phase of } \\
1^{\text {st }} \text { stage duration } \\
\text { (in minutes) }\end{array}$ & $\begin{array}{l}\text { Cervical dilatation } \\
\text { rate } \\
\text { (cm per hour) }\end{array}$ & $\begin{array}{l}\text { Total duration of } \\
\text { labor } \\
\text { (in hours) }\end{array}$ \\
\hline \multirow{5}{*}{$\begin{array}{l}\text { Camylofin } \\
75 \text { mg }(50+25) \\
\text { Primigravida } \\
\text { Vs Multigravida }\end{array}$} & $\begin{array}{l}\text { Primigravida }(\mathrm{N}=11) \\
\text { Mean }( \pm \mathrm{SD})\end{array}$ & $90.4( \pm 39.73)$ & $3.9( \pm 1.03)$ & $1.9( \pm 0.47)$ \\
\hline & $\begin{array}{l}\text { Multigravida }(\mathrm{N}=24) \\
\text { Mean }( \pm \mathrm{SD})\end{array}$ & $82.0( \pm 37.37)$ & $4.2( \pm 1.19)$ & $1.8( \pm 0.60)$ \\
\hline & $\begin{array}{l}\text { Mean difference } 95 \% \\
\text { C.I }\end{array}$ & $-21.29-38.28$ & $-1.20-0.44$ & $-0.28-0.49$ \\
\hline & SE & 13.9 & 0.4 & 0.2 \\
\hline & $\mathrm{P}$ value & 0.5570 & 0.3431 & 0.5808 \\
\hline $\begin{array}{l}\text { Camylofin } \\
100 \text { mg }(50+50) \\
\text { Primigravida } \\
\text { Vs Multigravida }\end{array}$ & \multicolumn{4}{|c|}{ No patient data for $100 \mathrm{mg}(50+50)$ primigravida } \\
\hline
\end{tabular}

Table 5: Primigravida vs Multigravida across the treatment arms.

\begin{tabular}{|c|c|c|c|c|c|}
\hline $\begin{array}{l}\text { Camylofin } \\
\text { dosage arm }\end{array}$ & Gravida & Statistical variable & $\begin{array}{l}\text { Active phase } \\
\text { of 1st stage } \\
\text { duration } \\
\text { (in minutes) }\end{array}$ & $\begin{array}{l}\text { Cervical } \\
\text { dilatation rate } \\
\text { (cm per hour) }\end{array}$ & $\begin{array}{l}\text { Total duration } \\
\text { of labor } \\
\text { (in hours) }\end{array}$ \\
\hline \multirow{5}{*}{$\begin{array}{l}50 \mathrm{mg} \\
\text { vs } \\
75 \mathrm{mg}(50+25)\end{array}$} & \multirow{5}{*}{ Primigravida } & $\begin{array}{l}50 \mathrm{mg}(\mathrm{N}=69) \\
\text { mean }( \pm \mathrm{SD})\end{array}$ & $\begin{array}{l}201.4 \\
( \pm 100.24)\end{array}$ & $2.3( \pm 1.16)$ & $3.4( \pm 2.07)$ \\
\hline & & $\begin{array}{l}75 \mathrm{mg}(50+25)(\mathrm{N}=11) \\
\text { Mean }( \pm \mathrm{SD})\end{array}$ & $90.4( \pm 39.73)$ & $3.9( \pm 1.03)$ & $1.9( \pm 0.47)$ \\
\hline & & $\begin{array}{l}\text { Mean difference } \\
95 \% \text { C.I }\end{array}$ & $76.44-145.46$ & $-2.31--0.85$ & $0.93-2.08$ \\
\hline & & $\mathrm{SE}$ & 30.7 & 0.4 & 0.6 \\
\hline & & $P$ value & $<0.0001$ & 0.0004 & $<0.0001$ \\
\hline \multirow{5}{*}{$\begin{array}{l}50 \mathrm{mg} \\
\text { vs } \\
75 \mathrm{mg}(50+25)\end{array}$} & \multirow{5}{*}{ Multigravida } & $\begin{array}{l}50 \mathrm{mg}(\mathrm{N}=93) \\
\text { Mean }( \pm \mathrm{SD})\end{array}$ & $142.3( \pm 85.48)$ & $3.1( \pm 1.71)$ & $2.9( \pm 2.72)$ \\
\hline & & $\begin{array}{l}75 \mathrm{mg}(50+25)(\mathrm{N}=24) \\
\text { Mean }( \pm \mathrm{SD})\end{array}$ & $82.0( \pm 37.37)$ & $4.2( \pm 1.19)$ & $1.8( \pm 0.60)$ \\
\hline & & $\begin{array}{l}\text { Mean difference } \\
95 \% \text { C.I }\end{array}$ & $37.13-83.62$ & $\begin{array}{l}-1.72- \\
-0.51\end{array}$ & $0.46-1.68$ \\
\hline & & $\mathrm{SE}$ & 17.9 & 0.4 & 0.6 \\
\hline & & $\mathrm{P}$ value & $<0.0001$ & 0.0005 & 0.0007 \\
\hline $\begin{array}{l}\mathbf{5 0} \mathrm{mg} \\
\text { vs } 100 \mathrm{mg} \\
(\mathbf{5 0 + 5 0 )}\end{array}$ & Primigravida & \multicolumn{4}{|c|}{ No patient in $100 \mathrm{mg}(50+50)$ group } \\
\hline \multirow{5}{*}{$\begin{array}{l}50 \mathrm{mg} \\
\mathrm{vS} \\
\mathbf{1 0 0} \mathbf{~ m g}(\mathbf{5 0 + 5 0})\end{array}$} & \multirow{5}{*}{ Multigravida } & $\begin{array}{l}50 \mathrm{mg}(\mathrm{N}=93) \\
\text { Mean }( \pm \mathrm{SD})\end{array}$ & $142.3( \pm 85.48)$ & $3.1( \pm 1.71)$ & $2.9( \pm 2.72)$ \\
\hline & & $\begin{array}{l}100 \mathrm{mg}(50+50)(\mathrm{N}=13) \\
\text { Mean }( \pm \mathrm{SD})\end{array}$ & $118.7( \pm 19.59)$ & $2.9( \pm 0.35)$ & $2.5( \pm 0.30)$ \\
\hline & & $\begin{array}{l}\text { Mean difference } \\
95 \% \text { C.I }\end{array}$ & $2.89-44.24$ & $-0.14-0.66$ & $-0.20-0.96$ \\
\hline & & SE & 23.9 & 0.5 & 0.8 \\
\hline & & $P$ value & 0.0260 & 0.1967 & 0.1966 \\
\hline $\begin{array}{l}75 \mathrm{mg}(50+25) \\
\text { vs } \\
100 \mathrm{mg}(50+50)\end{array}$ & Primigravida & \multicolumn{4}{|c|}{ No patient in $100 \mathrm{mg}(50+50)$ group } \\
\hline \multirow{2}{*}{$\begin{array}{l}75 \mathrm{mg}(50+25) \\
\text { vs } \\
\mathbf{1 0 0} \mathrm{mg}(\mathbf{5 0}+\mathbf{5 0})\end{array}$} & \multirow[t]{2}{*}{ Multigravida } & $\begin{array}{l}75 \mathrm{mg}(50+25) \\
(\mathrm{N}=24) \\
\text { Mean }( \pm \mathrm{SD})\end{array}$ & $81.9( \pm 37.37)$ & $4.2( \pm 1.19)$ & $1.8( \pm 0.60)$ \\
\hline & & $\begin{array}{l}100 \mathrm{mg}(50+50) \\
(\mathrm{N}=13)\end{array}$ & $118.7( \pm 19.59)$ & $2.9( \pm 0.35)$ & $2.5( \pm 0.30)$ \\
\hline
\end{tabular}

Continued. 


\begin{tabular}{|c|c|c|c|c|c|}
\hline $\begin{array}{l}\text { Camylofin } \\
\text { dosage arm }\end{array}$ & Gravida & Statistical variable & $\begin{array}{l}\text { Active phase } \\
\text { of 1st stage } \\
\text { duration } \\
\text { (in minutes) }\end{array}$ & $\begin{array}{l}\text { Cervical } \\
\text { dilatation rate } \\
\text { (cm per hour) }\end{array}$ & $\begin{array}{l}\text { Total duration } \\
\text { of labor } \\
\text { (in hours) }\end{array}$ \\
\hline & & \multicolumn{4}{|l|}{ Mean $( \pm$ SD $)$} \\
\hline & & Mean difference $95 \%$ C.I & $-55.83--17.8$ & $0.85-1.92$ & $-0.99--0.39$ \\
\hline & & SE & 11.2 & 0.3 & 0.2 \\
\hline & & $\mathrm{P}$ value & 0.0004 & $<0.0001$ & $<0.0001$ \\
\hline
\end{tabular}

Table 6: Reported AEs as coded using MedDRA by SOC, HLT, and PT across the three Camylofin dosage arms

\begin{tabular}{|c|c|c|c|c|}
\hline $\begin{array}{l}\text { MedDRA Coding } \\
\text { SOC, HLT, PT }\end{array}$ & $\begin{array}{l}50 \mathrm{mg}(\%) \\
(\mathrm{N}=162)\end{array}$ & $\begin{array}{l}75 \mathrm{mg}(50+25)(\%) \\
(\mathrm{N}=35)\end{array}$ & $\begin{array}{l}100 \mathrm{mg}(50+50)(\%) \\
(\mathrm{N}=13)\end{array}$ & $\begin{array}{l}\text { All Subjects }(\%) \\
(\mathbf{N}=210)\end{array}$ \\
\hline Maternal AEs & $23(14.8)$ & $12(34.3)$ & $9(69.2)$ & $44(21.0)$ \\
\hline $\begin{array}{l}\text { General disorders and } \\
\text { administration site conditions }\end{array}$ & $15(9.2)$ & $6(17.1)$ & $4(30.8)$ & $25(11.9)$ \\
\hline $\begin{array}{l}\text { Mucosal findings abnormal } \\
\text { Mucosal dryness }\end{array}$ & $13(8.0)$ & $6(17.1)$ & $4(30.8)$ & $23(10.9)$ \\
\hline $\begin{array}{l}\text { Febrile disorders } \\
\text { Post procedural fever }\end{array}$ & $2(1.2)$ & $0(0.0)$ & $0(0.0)$ & $2(0.9)$ \\
\hline $\begin{array}{l}\text { Gastrointestinal disorders } \\
\text { Nausea and vomiting symptoms } \\
\text { Vomiting }\end{array}$ & $13(8.0)$ & $5(14.3)$ & $5(38.5)$ & $23(10.9)$ \\
\hline $\begin{array}{l}\text { Injury, poisoning and } \\
\text { procedural complications } \\
\text { Neurological and psychiatric } \\
\text { procedural complications } \\
\text { Compensatory sweating }\end{array}$ & $1(0.6)$ & $0(0.0)$ & $0(0.0)$ & $1(0.5)$ \\
\hline $\begin{array}{l}\text { Pregnancy, puerperium and } \\
\text { perinatal conditions }\end{array}$ & $6(3.7)$ & $3(8.6)$ & $1(7.7)$ & $10(4.7)$ \\
\hline $\begin{array}{l}\text { Maternal complications of } \\
\text { pregnancy NEC } \\
\text { Inferior vena cava syndrome }\end{array}$ & $4(2.5)$ & $(0.0)$ & $(0.0)$ & $4(1.9)$ \\
\hline $\begin{array}{l}\text { Postpartum complications NEC } \\
\text { Postpartum hemorrhage }\end{array}$ & $2(1.2)$ & $3(8.6)$ & $1(7.7)$ & $6(2.8)$ \\
\hline Neonatal AEs & $6(3.7)$ & 0 & 0 & $6(2.9)$ \\
\hline $\begin{array}{l}\text { Respiratory, thoracic and } \\
\text { mediastinal disorders } \\
\text { Neonatal hypoxic conditions } \\
\text { Neonatal asphyxia }\end{array}$ & $2(1.2)$ & $(0.0)$ & $(0.0)$ & $2(0.9)$ \\
\hline $\begin{array}{l}\text { Pregnancy, puerperium and } \\
\text { perinatal conditions }\end{array}$ & \multirow[b]{2}{*}{$4(2.5)$} & \multirow[b]{2}{*}{$0(0.0)$} & \multirow[b]{2}{*}{$0(0.0)$} & \multirow[b]{2}{*}{$4(1.9)$} \\
\hline $\begin{array}{l}\text { Amniotic fluid and cavity } \\
\text { disorders of pregnancy NEC } \\
\text { Meconium stain }\end{array}$ & & & & \\
\hline
\end{tabular}

Table 7: Distribution of patient ADRs is given across treatment arms using medical dictionary, MedDRA coding (SOC, HLT, PT).

\begin{tabular}{|c|c|c|c|c|}
\hline $\begin{array}{l}\text { MedDRA coding } \\
\text { SOC, HLT, PT }\end{array}$ & $\begin{array}{l}50 \mathrm{mg}(\%) \\
(\mathrm{N}=162)(\%)\end{array}$ & $\begin{array}{l}75 \mathrm{mg}(50+25) \\
(\%) \\
(\mathrm{N}=35)\end{array}$ & $\begin{array}{l}100 \mathrm{mg} \\
(50+50)(\%) \\
(\mathrm{N}=13)\end{array}$ & $\begin{array}{l}\text { All subjects }(\%) \\
(\mathrm{N}=\mathbf{2 1 0})\end{array}$ \\
\hline \multicolumn{5}{|c|}{ Number of subjects with at least one ADRs } \\
\hline Maternal & $21(12.9)$ & $11(31.4)$ & $6(46.1)$ & $38(18.1)$ \\
\hline Neonatal & $3(1.8)$ & $0(0.0)$ & $0(0.0)$ & $3(1.4)$ \\
\hline \multicolumn{5}{|l|}{ Maternal ADR } \\
\hline $\begin{array}{l}\text { Skin and subcutaneous tissue } \\
\text { disorders }\end{array}$ & $10(6.2)$ & $2(5.7)$ & $1(7.7)$ & $13(6.2)$ \\
\hline
\end{tabular}

Continued. 


\begin{tabular}{|c|c|c|c|c|}
\hline $\begin{array}{l}\text { MedDRA coding } \\
\text { SOC, HLT, PT }\end{array}$ & $\begin{array}{l}50 \mathrm{mg}(\%) \\
(\mathrm{N}=162)(\%)\end{array}$ & $\begin{array}{l}75 \mathrm{mg}(50+25) \\
(\%) \\
(\mathrm{N}=35)\end{array}$ & $\begin{array}{l}100 \mathrm{mg} \\
(\mathbf{5 0 + 5 0 )}(\%) \\
(\mathrm{N}=13)\end{array}$ & $\begin{array}{l}\text { All subjects }(\%) \\
(\mathrm{N}=\mathbf{2 1 0})\end{array}$ \\
\hline $\begin{array}{l}\text { Rashes, eruptions and exanthems } \\
\text { NEC }\end{array}$ & \multirow[t]{2}{*}{$0(0.0)$} & \multirow[t]{2}{*}{$2(5.7)$} & \multirow[t]{2}{*}{$1(7.7)$} & \multirow[t]{2}{*}{$3(1.4)$} \\
\hline Skin rashes & & & & \\
\hline Probable & $0(0.0)$ & $2(5.7)$ & $1(7.7)$ & $3(1.4)$ \\
\hline Possible & $0(0.0)$ & $0(0.0)$ & $0(0.0)$ & $0(0.0)$ \\
\hline $\begin{array}{l}\text { Skin vasomotor conditions } \\
\text { Flushing }\end{array}$ & $10(6.2)$ & $0(0.0)$ & $0(0.0)$ & $10(4.8)$ \\
\hline Probable & $6(3.7)$ & $0(0.0)$ & $0(0.0)$ & $6(2.9)$ \\
\hline Possible & $4(2.5)$ & $0(0.0)$ & $0(0.0)$ & $4(1.9)$ \\
\hline $\begin{array}{l}\text { Injury, poisoning and procedural } \\
\text { complications }\end{array}$ & \multirow{3}{*}{$2(1.2)$} & \multirow{3}{*}{$0(0.0)$} & \multirow{3}{*}{$0(0.0)$} & \multirow{3}{*}{$2(0.9)$} \\
\hline $\begin{array}{l}\text { Neurological and psychiatric } \\
\text { procedural complications }\end{array}$ & & & & \\
\hline Compensatory sweating & & & & \\
\hline Probable & $2(1.2)$ & $0(0.0)$ & $0(0.0)$ & $2(0.9)$ \\
\hline Possible & $0(0.0)$ & $0(0.0)$ & $0(0.0)$ & $0(0.0)$ \\
\hline $\begin{array}{l}\text { General disorders and } \\
\text { administration site conditions }\end{array}$ & $16(9.9)$ & $6(17.1)$ & $4(30.8)$ & $26(12.4)$ \\
\hline Mucosal findings abnormal & $14(8.6)$ & $6(17.1)$ & $4(30.8)$ & $24(11.4)$ \\
\hline Probable & $14(8.6)$ & $6(17.1)$ & $4(30.8)$ & $24(11.4)$ \\
\hline Possible & $0(0.0)$ & $0(0.0)$ & $0(0.0)$ & $0(0.0)$ \\
\hline $\begin{array}{l}\text { Febrile disorders } \\
\text { Post procedural fever }\end{array}$ & $2(1.2)$ & $0(0.0)$ & $0(0.0)$ & $2(0.9)$ \\
\hline Probable & $2(1.2)$ & $0(0.0)$ & $0(0.0)$ & $2(0.9)$ \\
\hline Possible & $0(0.0)$ & $0(0.0)$ & $0(0.0)$ & $0(0.0)$ \\
\hline Nervous system disorders & \multirow{3}{*}{$0(0.0)$} & \multirow{3}{*}{$2(5.7)$} & \multirow{3}{*}{$1(7.7)$} & \multirow{3}{*}{$3(1.4)$} \\
\hline Headaches NEC & & & & \\
\hline Headache & & & & \\
\hline Probable & $0(0.0)$ & $2(5.7)$ & $1(7.7)$ & $3(1.4)$ \\
\hline Possible & $0(0.0)$ & $0(0.0)$ & $0(0.0)$ & $0(0.0)$ \\
\hline Gastro-intestinal disorders & \multirow[b]{2}{*}{$2(1.2)$} & \multirow[b]{2}{*}{$1(2.9)$} & & \\
\hline $\begin{array}{l}\text { Nausea and vomiting symptoms } \\
\text { Vomiting }\end{array}$ & & & $0(0.0)$ & $3(1.4)$ \\
\hline Probable & $1(0.6)$ & $1(2.9)$ & $0(0.0)$ & $2(0.9)$ \\
\hline Possible & $1(0.6)$ & $0(0.0)$ & $0(0.0)$ & $1(0.5)$ \\
\hline Neonatal & $3(1.8)$ & $0(0.0)$ & $0(0.0)$ & $3(1.4)$ \\
\hline $\begin{array}{l}\text { Respiratory, thoracic and } \\
\text { mediastinal disorders }\end{array}$ & & & & \\
\hline $\begin{array}{l}\text { Neonatal hypoxic conditions } \\
\text { Neonatal asphyxia }\end{array}$ & $2(1.2)$ & $0(0.0)$ & $0(0.0)$ & $2(0.9)$ \\
\hline Probable & $1(0.6)$ & $0(0.0)$ & $0(0.0)$ & $1(0.5)$ \\
\hline Possible & $1(0.6)$ & $0(0.0)$ & $0(0.0)$ & $1(0.5)$ \\
\hline $\begin{array}{l}\text { Pregnancy, puerperium and } \\
\text { perinatal conditions }\end{array}$ & & & & \\
\hline $\begin{array}{l}\text { Amniotic fluid and cavity disorders } \\
\text { of pregnancy NEC }\end{array}$ & $1(0.6)$ & $0(0.0)$ & $0(0.0)$ & $1(0.5)$ \\
\hline Meconium stain & & & & \\
\hline Probable & $1(0.6)$ & $0(0.0)$ & $0(0.0)$ & $1(0.5)$ \\
\hline Possible & $0(0.0)$ & $0(0.0)$ & $0(0.0)$ & $0(0.0)$ \\
\hline
\end{tabular}

Multigravida patients were comparatively higher in $50 \mathrm{mg}$ $(57.4 \%)$ and $75 \mathrm{mg}(50+25)(68.6 \%)$ dose categories than the primigravida. However, in $100 \mathrm{mg}(50+50)$ category, there were no primigravida patients. The mean cervical 
dilatation rate (cm per hour) is comparable between $50 \mathrm{mg}$ $(2.8 \mathrm{~cm} / \mathrm{hr})$ and $100 \mathrm{mg}(50+50)(2.9 \mathrm{~cm} / \mathrm{hr})$ dose categories. The rate is slightly increased in $75 \mathrm{mg}(50+25)$ dose category $(4.1 \mathrm{~cm} / \mathrm{hr})$, which is higher than the overall average rate of dilatation $(3.0 \mathrm{~cm} / \mathrm{hr})$.

The mean cervical effacement rate was $18.0 \%$ per hour in $50 \mathrm{mg}$ and $27.1 \%$ per hour in $75 \mathrm{mg}(50+25)$ and $100 \mathrm{mg}$ $(50+50)$ arms, which is higher by about $50 \%$.

The mean Apgar score for the treatment arm $50 \mathrm{mg}$ was 8.6 (1 min) and 9.3 (5 min). The mean Apgar scores at 1 and 5 minutes were almost the same for $75 \mathrm{mg}(50+25)$, 9.9 and 9.9, and $100 \mathrm{mg}(50+50)$ arms, 10.0 and 10.0 , respectively. The overall score was good for $1 \mathrm{~min}(8.9)$ and 5 min (9.5) with no noted complications.

No obvious differences with means of maternal age, gravida, gestational age and Apgar score were observed across the three dose categories.

\section{Efficacy}

The significance analysis of three treatment arms for exploratory parameters was analyzed using independent $t$ test (two arms) and ANOVA test (three arms). The resulted mean with $95 \%$ C.I and p-values are given in Tables 2 and 3.

In the significance analysis of overall groups, 50 versus 75 $\mathrm{mg}(50+25)$ and $75 \mathrm{mg}(50+25)$ versus $100 \mathrm{mg}(50+50)$ were resulted as statistically significant ( $p$-value $<0.0003$ ) for all three endpoints, duration of active phase, cervical dilatation rate and total duration of labor.

While, in group 50 versus $100 \mathrm{mg}(50+50)$, only mean duration of active phase and mean total duration of labor were statistically significant ( $p$-value $<0.005)$ and mean cervical dilatation rate was not statistically significant ( $\mathrm{p}$ value 0.5349 ).

The ANOVA test for three arms wise came significant (pvalue $<0.01)$ for 50 versus $75 \mathrm{mg}(50+25)$ mean duration of active phase, mean cervical dilatation rate, and mean total duration of labor. While dose increase from 50 to 100 $\mathrm{mg}(50+50)$ was not significant for all three parameters (pvalue >0.05). For $75 \mathrm{mg}(50+25)$ versus $100 \mathrm{mg}(50+50)$, only rate of cervical dilatation was significant (p-value $<0.05)$ whereas the other two parameters were not significant ( $\mathrm{p}$-value $>0.05$ ).

\section{Primigravida versus multigravida analysis}

The significance analysis of primigravida versus multigravida within the treatment arms is shown in Table 4 and across the treatment arms is given in Table 5.

\section{Within the treatment arms}

In dose category, $50 \mathrm{mg}$, the mean duration of active phase and mean cervical dilatation rate were significant $((\mathrm{p}$-value
$<0.0003$ ) between primigravida and multigravida whereas mean total duration of labor was not significant (Table 4). For dose arm $75 \mathrm{mg}(50+25)$, none of the parameters were significant (p-value >0.05). While in $100 \mathrm{mg}(50+50)$ category there were no primigravida patients to compare with multigravida patients.

The mean cervical dilatation rate was increased by $0.8 \mathrm{~cm}$, mean duration of active phase was reduced by $60 \mathrm{mins}$ and mean total duration of labor was reduced by 0.5 hours from primigravid to multigravida in $50 \mathrm{mg}$ arm. However, the change was not very considerable for $75 \mathrm{mg}(50+25)$ with $0.3 \mathrm{~cm}, 8 \mathrm{mins}$, and 0.1 hours, respectively.

\section{Across the treatment arms}

For dosage groups, $50 \mathrm{mg}$ versus $75 \mathrm{mg}(50+25)$, both primigravida and multigravida and group $75 \mathrm{mg}(50+25)$ versus $100 \mathrm{mg}(50+50)$, multigravida, the differences between mean duration of active phase, mean cervical dilatation rate and mean total duration of labor were significant (p-value <0.001) (Table 5). For group $50 \mathrm{mg}$ versus $100 \mathrm{mg}(50+50)$ multigravida, only mean duration of active phase of labor was significant ( $p$-value $<0.05$ ) and the other two parameters were not significant. There were no primigravida patients in $100 \mathrm{mg}(50+50)$ arm to compare the significance with the other two groups.

\section{Safety}

Safety assessments consist of all AE and/or ADRs reported across the three Camylofin dosage arms captured during post-delivery follow-up visits. AE analyses were performed for all patients who received at least one dose of Camylofin injection during the maintenance therapy. AEs were broken down as maternal (anticholinergic (dryness of mouth, fever, and sweating), hypotension, PPH and vomiting) and neonatal (birth asphyxia and meconium stained liquor). The results from the safety analysis of the treatment arms are provided in Table 6.

Overall, 68 AEs, 62 maternal and 6 neonatal, were documented for 44 patients $(21.0 \%)$ and 6 neonates $(2.9 \%)$ across all three treatment arms. Of these total AEs, no serious $\mathrm{AE}$ (SAE) and treatment emerging (TE) deaths were reported (Table 6). Amongst, $69.2 \%$ was reported in $100 \mathrm{mg}(50+50) \mathrm{arm}$, followed by $34.3 \%$ in $75 \mathrm{mg}(50+25)$ and $14.8 \%$ in $50 \mathrm{mg}$. While neonatal events were reported only in $50 \mathrm{mg}$ arm.

All ADRs were assessed with respect to their causal relationship to study medication, i.e. 'related' or 'not related' to the administration of Camylofin dihydrochloride injection. The five categories used to assess the causal relationship in the safety database (probable, possible, probably not, not related, not assessable). ADRs assessed as related to occurring in patients in all three arms, broken down by MedDRA SOC, HLT and PT are presented in Table 7. Total 38 patients (18.1\%) were reported maternal ADRs wherein $50 \mathrm{mg}$ 
arm, 7 patients had reported 2 ADRs and 2 patients reported 3 ADRs. Two patients in $50 \mathrm{mg}$ arm had both maternal ADR and neonatal ADR.

The incidences of AEs were $14.8 \%(\mathrm{n}=23)$ in $50 \mathrm{mg}$ arm, $34.3 \%(\mathrm{n}=12)$ in $75 \mathrm{mg}(50+25)$ arm and $69.0 \%(\mathrm{n}=9)$ in $100 \mathrm{mg}(50+50) \mathrm{arm}$. Of the total 68 AE events, 34 events $(50.0 \%)$, as catered to maternal - dryness of mouth, fever, sweating and vomiting, and neonatal - birth asphyxia and meconium stain, were related to administration of Camylofin dihydrochloride injection, i.e. ADRs.

Out of the total 52 ADRs (34 AEs + 18 ADRs), 49 were reported for maternal and 3 for neonates. The causality assessment of these ADRs had suggested that 46 ADRs, (44 maternal and 2 neonate) had 'probable' and 6 ADRs (5 maternal and 1 neonate) had 'possible' relation with the Camylofin injection.

Mucosal dryness as underlying disease was the leading AE in the total cases of the study patients followed by vomiting, postpartum hemorrhage, inferior vena cava syndrome, post-partum fever, and compensatory sweating in at least 1 case. While for neonates, meconium stain and neonatal asphyxia were the commonly reported events.

\section{DISCUSSION}

Camylofin dihydrochloride injection was studied in 210 pregnant women in labor to collect clinical and obstetric data that represents the real-world clinical practice to the extent possible.

Primary objective is to determine the clinical and obstetric characteristics of women in active phase of labor who were managed with Camylofin injection and secondary objective is to evaluate the tolerability profile of the patients treated with the injection. The exploratory objectives are to evaluate the comparative effectiveness of different doses of Camylofin injection in mean duration of active phase of 1st stage of labor, mean cervical dilatation rate and mean total duration of labor in primigravida and multigravida patients.

Overall data from the study indicated that Camylofin injections has been a drug of choice in managing the active phase of labor by accelerating cervical dilatation for both primigravida and multigravida patients. Also, dose escalation of the injection was considered to a moderate extent in multigravida patients.

The $50 \mathrm{mg}$ dose of Camylofin injection was sufficient enough to bring in the necessary cervical dilatation in 162 patients $(77.1 \%)$ whereas 35 patients $(16.7 \%)$ needed additional dose, i.e., $25 \mathrm{mg}$ of Camylofin injection and 13 patients $(6.2 \%)$ required the same dose for second time, i.e., $50 \mathrm{mg}$ of Camylofin injection. Overall, irrespective of the gravidity, the dose escalation from $50 \mathrm{mg}$ to $75 \mathrm{mg}$ $(50+25)$ was effective in accelerating the labor with increase in the rate of cervical dilatation. At the same time, further increase in dose to $100 \mathrm{mg}(50+50)$ does not appear to be significant with respect to cervical dilatation rate and in turn reducing the total duration of labor time.

Camylofin has been reported to possess neurotropic action via anti-cholinergic effect and hence known to cause anticholinergic effects (dryness of mouth, fever, sweating), besides other side effects like hypotension, PPH, vomiting and maternal tachycardia. In the current study none of the study population had reported tachycardia in mother and/or neonate. The symptoms associated with the higher dose, specifically dry mouth and rashes, were (percentage wise) high in two injection groups, $100 \mathrm{mg}(50+50)$ and $75 \mathrm{mg}(50+25)$, than the single injection, $50 \mathrm{mg}$ (Table 6 and 7). The APGAR score was normal for all neonates. In all three arms, comparable patterns of AEs as well as ADRs were presented, which is mainly consistent with the safety profile known for the population examined, including the occurrence of rare cases of severe events. Moreover, ADRs found in this study were already known and reported for Camylofin dihydrochloride injection in management of acceleration of labor. Other known side effects such as severe cases of infusion-related events or allergic hypersensitivity reactions were not observed in the current study. Even though the pattern of events was comparable among all three arms patients, the AE incidence was comparatively high in $100 \mathrm{mg}(50+50)$ arm, then $75 \mathrm{mg}(50+25)$ arm than $50 \mathrm{mg}$ arm. No treatment emerging death, serious adverse event and death case in patients, were reported during the study period. The outcomes of the study are compared with the values reported in the literature. All of the reported studies had used single Camylofin injection $(50 \mathrm{mg})$ and hence the corresponding values from the current study were only compared to assess the results.

In a placebo-controlled study, the reported mean rate of cervical dilatation was $1.92 \mathrm{~cm} /$ hour and the mean total duration was 4 hours 42 minutes in $50 \mathrm{mg}$ Camylofin dose. ${ }^{14}$ In comparator-studies, a single-center study where Camylofin injection (50 mg) was compared in 100 pregnant women, mean duration of active phase of $1 \mathrm{st}$ stage of labor was $141.4( \pm 55.41)$ minutes and the mean rate of cervical dilatation was $3.3( \pm 1.03) \mathrm{cm} /$ hours and mean duration of delivery was $172.0( \pm 60.82)$ minutes. ${ }^{1}$ In another randomized comparative study conducted on 63 primigravida women, the cervical dilatation rate was 1.78 $\mathrm{cm} /$ hour and mean duration of active phase of 1st stage was 332.5 minutes with $50 \mathrm{mg}$ Camylofin injection. ${ }^{14} \mathrm{~A}$ randomized controlled study, 50 primigravida women, the cervical dilatation rate with $50 \mathrm{mg}$ Camylofin injection was, $3.14 \mathrm{~cm} /$ hour. $^{14}$ In the current study, the corresponding values $(50 \mathrm{mg})$ are $167.5( \pm 96.32)$ minutes and $2.8( \pm 1.55) \mathrm{cm} /$ hour, $186.0( \pm 148.2)$ minutes (3 hours 6 minutes), respectively. There are no reported studies using multiple (two) injections of Camylofin drug (in the Indian population) and therefore, no comparisons are drawn with any literature reports. 


\section{Limitations}

The gravida analysis included groups with different sample sizes (e.g. Multigravida patients in $50 \mathrm{mg}-93$ patients versus $100 \mathrm{mg}-13$ patients), for this reason, the evaluation and the reported p-values across the groups limited to the respective groups. The results from within the groups, $50 \mathrm{mg}$, primigravida versus multigravida and $75 \mathrm{mg}(50+25)$, primigravida versus multigravida can be considered owing to comparable sample sizes. However, within the group analysis would not offer information about the effectiveness of escalation of dose from $50 \mathrm{mg}$ to $50+25 \mathrm{mg}$ or $50+50 \mathrm{mg}$. Importantly, no primigravida patients in the $100 \mathrm{mg}(50+50)$ dosage arm further limits the extendibility of the resulted gravida analysis.

Considering all the above reasons, drawing the gravida analysis observations and inferences, if any, would rather be taken with extra caution particularly for $100 \mathrm{mg}$ $(50+50)$ dose

\section{CONCLUSION}

The study infers Camylofin injection usage with consideration of dose escalation in the routine clinical management of labor. Both mother and neonate had shown comparable tolerability profile across the three dosage arms with minimal events.

\section{ACKNOWLEDGMENTS}

Acknowledge the support and guidance of Lavina Yadav, Head of Real-World Evidence, Sekhmet Technologies (THB) and Mohini Gahlot, Clinical Research Coordinator, Sekhmet Technologies (THB) for support in MedDRA coding. THB provided medical writing support.

\section{Funding: No funding sources}

Conflict of interest: None declared

Ethical approval: The study was approved by the Institutional Ethics Committee

\section{REFERENCES}

1. Sarbhjit K, Bajwa SK, Parmjit K, Surinder B. To compare the effect of camylofin dihydrochloride (Anafortin) with combination of valethamate bromide (epidosin) and hyoscine butyl-n-bormide (buscopan) on cervical dilation. J Clin Diagn Res. 2013;7:189799.

2. Cunningham FG, Leveno KJ, Bloom SL, Hauth JC, Rouse DJ, Spong CY. Parturition. Williams Obstetrics. 23rd Edition, New York: McGraw Hill Medical 2010;136-60.

3. Nyfløt LT, Stray-Pedersen B, Forsén L, Vangen S. Duration of labor and the risk of severe postpartum hemorrhage: A case-control study. PLoS ONE. 2017;12:e0175306.

4. Ranjana, Sinha A. Incidence, causes and fetomaternal outcomes of obstructed labour in a tertiary health care centre. Int J Reprod Contracept Obstet Gynecol. 2017;6:2817-21.

5. Say L, Chou D, Gemmill A, Tuncalp O, Moller AB, Daniels J, et al. Global causes of maternal death: a WHO systematic analysis. Lancet Glob Health. 2014;2:e323-33.

6. Roos N, von Xylander SR. Why do maternal and newborn deaths continue to occur? Best Pract Res Clin Obstet Gynaecol. 2016;36:30-44.

7. Adhikari SM, Dasgupta M. Management of obstructed labour: A retrospective study. Journal Obs Gyna Ind. 2005;55(1):48-51.

8. Devi R, Andallu R, Laxmi DP, Sundari MT. A clinical study of prolonged pregnancies with regard to maternal and perinatal outcome. IOSR Journal of Dental and Medical Sciences (IOSR-JDMS). 2016;15:31-4.

9. Rohwer AC, Khondowe O, Young T. Antispasmodics for labour. Cochrane Database Syst Rev. 2013;6:CD009243.

10. Brown HC, Paranjothy S, Dowswell T, Thomas J. Package of care for active management in labour for reducing caesarean section rates in low-risk women. Cochrane Database Syst Rev. 2013;9:CD004907.

11. Leppert PC. Anatomy and physiology of cervical ripening. Clin Obstet Gynecol 1995;38:267-79.

12. Buhimschi CS, Buhimschi IA, Malinow AM, Saade GR, Garfield RE, Weiner CP. The forces of labour. Fetal and Maternal Medicine Review 2003;14:273307.

13. Samuels L. Pharmacotherapy update: hyoscine butylbromide in the treatment of abdominal spasms. Clinical Medicine Insights: Therapeutics 2009;1:64755.

14. Mayadeo N, Gangadhar A, Das S. Camylofin in the management of prolonged labor: a review of evidence. Int J Reprod Contracept Obstet Gynecol. 2017;6:776-780.

15. Warke HS, Chauhan AR, Raut VERSUS, Ingle KM. Effectivness of camylofin dihydrochloride in acceleration of labour. A randomised double blind trial. Bombay Hospital J. 2003;45:420-423.

16. Dayama SS, Patil SS, Sambarey PW. A randomised controlled study of intramuscular camylofin dihydrochloride versus intravenous hyoscine butylbromide in augmentation of labour. Global J Med Res: Gynecol Obstet. 2016;16:1-6.

17. Rajani U, Binu P. A randomized comparative study of intramuscular camylofin dihydrochloride and intravenous drotaverine hydrochloride on cervical dilatation in labor. Indian $\mathbf{J}$ Clinical Practice. 2015;26:558-563.

18. Brock N. Pharmacology of Avacan. Dtsch Med Wochenschr. 1951;76:474-477.

19. Murano T, Yamane K. A study of metabolism of Isoamyl-alpa-N(betadiethylaminoethyl)Aminophenylacetate - (Avacan). Jpn J Pharmacol. 1956;5:122-131.

20. Daftary SN, Desai SV. Selected Topics in Obstetrics and Gynaecology-1 for Postgraduates and 
Practitioners. 1st Edition, New Delhi, Bi Publications Pvt Ltd. 2007.

21. Padubidri VG, Daftary SN. Hawkins and Bourne Shaw's Textbook of Gynecology E-Book. 16th Edition, New Delhi, Elsevier India Private Limited. 2015;472.

22. Salhan S, Textbook of Obstetrics. 2nd Edition, New Delhi, Jaypee Brothers Medical Publishers (P) Ltd. 2016; 250.

23. Malhotra N, Puri R, Malhotra J, Singh KN. Operative Obstetrics and Gynecology. New Delhi, Jaypee Brothers Medical Publishers (P) Ltd. 2014;185.

24. Pramod TK. Best Aid to Gynecology. First Edition, New Delhi, Jaypee Brothers Medical Publishers (P) Ltd. 2013;257.

25. Arvind Rao K. Textbook of Gynaecology. First Edition, New Delhi, Elsevier India Private Limited. $2008 ; 42$
26. Daftary SN, Desai SV, Thanawala U, Bhinde A, Levi J, Patki A, et al. Programmed Labor: Indegenous protocol to optimize labor outcome. South Asian Federation of Obstetrics and Gynecology. 2009;1:614.

27. Bachani S, Topden S. Active management of labor in a low-resource setting and its impact on cesarean section rates. Int J Gynaecol Obstet. 2006;94:54-5.

Cite this article as: Taneja VK, Telang MA. A retrospective, observational, real-world evidence study to understand the clinical and obstetric characteristics and tolerability profile of women in labor managed with camylofin dihydrochloride injection. Int J Reprod Contracept Obstet Gynecol 2021;10:1421-32. 